\title{
CLFP intrinsic constraints-based group management of blended learning situations
}

\author{
M. Pérez-Sanagustín, J. Burgos, D. Hernández-Leo, J. Blat \\ ICT Department, Universitat Pompeu Fabra, C/Roc Boronat 138, 08018 Barcelona, Spain \\ \{mar.perez, davinia.hernandez, javier.burgos, josep.blat\}@upf.edu.com
}

\begin{abstract}
When applying a Collaborative Learning Flow Pattern (CLFP) to structure sequences of activities in real contexts, one of the tasks is to organize groups of students according to the constraints imposed by the pattern. Sometimes, unexpected events occurring at runtime force this pre-defined distribution to be changed. In such situations, an adjustment of the group structures to be adapted to the new context is needed. If the collaborative pattern is complex, this group redefinition might be difficult and time consuming to be carried out in real time. In this context, technology can help on notifying the teacher which incompatibilities between the actual context and the constraints imposed by the pattern. This chapter presents a flexible solution for supporting teachers in the group organization profiting from the intrinsic constraints defined by a CLFPs codified in IMS Learning Design. A prototype of a web-based tool for the TAPPS and Jigsaw CLFPs and the preliminary results of a controlled user study are also presented as a first step towards flexible technological systems to support grouping tasks in this context.
\end{abstract}

Keywords Constraints, Flexibility, CLFP, Group management, IMS LD

\section{Introduction}

$\mathrm{S}$

CRIPTS are the computational solution proposed in the Computer-Supported Col-

laborative Learning (CSCL) field to guide and support potentially fruitful interactions in terms of learning benefits. Scripting a learning process means shaping interactions without spoiling the natural richness of free collaboration in order to produce situations of effective learning [3, 4]. However, when applying a script to a blended learning scenario - where online, technology supported and face to face (f2f) activities are combined in a given space - some unpredictable situations arising from the context force the scripts' constraints to be re-defined on the fly. One of the main aspects usually affected by this contextual variability is the group organization and the role distribution along the script's phases. When these situations occur, it is necessary to re-distribute groups of participants and roles in a 
flexible manner to adjust the script to the actual situation without violating its principles; i.e. the constraints that structure the collaboration. Different solutions and tools have been developed to provide support to collaborative practices $[6,9$, 14]. Nevertheless, these systems are still too rigid to capture the unexpected changes occurring in educational contexts and, in particular, in blended learning contexts. Specialized and interoperable tools are needed for supporting these flexibility demands.

This work proposes a flexible solution for managing groups of students according to the variability of the context and the intrinsic constraints stipulated by Collaborative Learning Flow Patterns (CLFPs) codified with the IMS LD specification. CLFPs capture the essence of well-known techniques for structuring the flow of learning activities to potentially produce effective learning from collaborative situations [5, 7]. Whereas, the IMS Learning Design (IMS LD) specification allows its formalization into a computer-interpretable design. Taking as a basis a constrain-based framework proposed by Dillenbourg and Tchounikine we analyze the flexibility requirements of two representative examples of complex CL (Collaborative Learning) activities: the TAPPs and Jigsaw CLFPs. With the results of the analysis we implement a Web-based prototype for flexibly supporting the group management both examples.

Section 2 discusses the concept of flexibility, presents some of the existing approaches for supporting the group management that inspired this work and gives an overview of the solution proposed. Section 3 presents the results of studying the intrinsic constrains for the TAPPS and the Jigsaw CLFPs and their representation in IMS LD. Section 4 explains the web-based prototype and its architecture. Finally, section 5 and 6 report the preliminary results obtained from a controlled user study, the main conclusions and future work.

\section{Flexible solutions for supporting CSCL scripts}

Using a script means to structure the learning flow and organize groups of students to constrain collaborative interactions. If these constraints are too strong, the script can spoil the natural richness of free collaboration; whereas if the constraints are too weak, the expected interactions might not be produced $[2,3,4]$. Consequently, the design of technological settings for supporting CSCL scripts must be sufficiently flexible for dealing with the main dimensions that arise from these two aspects. It must help to structure collaboration, but should also support some variability when applied into a real context. This section reviews some of the studies that inspired this work. In one hand, we discuss the concept of flexibility adopted as a basis for the solution proposed. On the other hand, we go through some approaches developed for supporting the group management in collaborative practices and highlight their limitations. Finally, we introduce our proposal for supporting teachers in the group organization and adaptation that will be developed in the next sections. 


\subsection{Flexibility as disjunction of intrinsic and extrinsic constraints}

Dillenbourg and Tchounikine (2007) support the idea that, due to the unpredictability of the script during the enactment phase, the teacher and the student must be able to modify some script features. Based on this, they propose a conceptual constraint-based framework that defines flexibility in terms of intrinsic and extrinsic constraints [4]. The intrinsic constraints arise from the principles from which the script has been generated and must be respected in order to get a fruitful collaboration. The extrinsic constraints arise from those elements induced by the technology of contextual factors (limitations in the number of students, evaluation elements ...). The dissociation of constraints proposed marks the boundaries of flexibility for the teacher and students, and provides the basis for a computational platform of interaction. This platform should be sufficiently flexible to maintain interaction patterns in the space of extrinsic constraints, without violating the intrinsic constraints in each of the phases of the script development process (edition, instantiation and enactment). As a conclusion, Dillenbourg and Tchounikine propose addressing the operationalization of CSCL scripts by handling multiple representations of the same script: the script to be executed; the current interaction patterns or emergent organization of teams; the intrinsic and extrinsic constraints that result respectively from the pedagogical design; and from the decision and the visual representations of the script for the students and teachers.

In this work, we adopt the dissociation between intrinsic and extrinsic constraints proposed in this constraint-based framework for delimiting our notion of flexibility and the scope of this work.

\subsection{Limitations in supporting group management in collaborative blended learning scenarios}

Several approaches have been developed for technologically supporting the group management in collaborative learning. However, and despite of their potential for solving some aspects of collaborative tasks, they lack on facing some of the problems arising when enacting collaborative learning flows in blended learning scenarios. Here we classify, describe and analyze some of these approaches under the idea of flexibility introduced in the previous section.

\section{Specialized grouping tools}

A study by Ounnas proposes a framework for learner group formation, based upon satisfying the constraints of the teacher by reasoning over semantic data about the potential participants $[13,14]$. As a technological support based on this framework, Ounnas proposes a tool that enables forming groups of students according 
to a set of constraints defined by the user and the semantic data that characterize the potential students participating in the activity. The result is a simple and powerful solution for easily allocating all students in groups. In the same line, an study by Hwang et al [9] proposes a genetic algorithm as a basis for an assistant system for organizing efficient cooperative groups that fit the learning objectives set by the instructor.

Despite of the potential of these approaches, they propose solutions for supporting the group organization for a particular activity and not for sequences of activities following a learning flow such as those defined by scripts. Thus, these solutions do not consider the relations established within group members from a set of interrelated activities, i.e. group formation according to the students roles in previous activities. Moreover, these applications do not assist the teacher in understanding the adaptation needs that emerge from the contextual situations and their relation with the intrinsic script constrains.

\section{Specialized grouping tools conforming with IMS LD}

One of the best-established modeling languages that are used to develop applications in educational contexts is IMS Learning Design (IMS LD) [10, 11, 12]. This specification enables the computational representation of learning flows according to a wide range of pedagogies in online learning. These computational learning flows are defined in different phases: learning flows are typically determined according to the educational objectives at design time, particularized to the specific learning situation at instantiation time and delivered to the participants as an activity to perform at enactment time. In CSCL, different approaches conform to IMS LD have been developed to support one of these phases. These computational representations are suitable to be interpreted by a compliant system as a way of alleviating teacher and learner management tasks.

As a support for the design time, Hernández-Leo et al propose an authoring tool for the edition of designs based on Collaborative Learning Flow Patterns conforming to IMS LD [8]. These patterns represent the techniques used to structure the flow of types of learning activities involved in collaborative learning situations. As a result, this tool provides the educator with a computational learning flow suitable to be interpreted by a system conformig to IMS LD that organizes groups of students within an activity sequence during the edition time, but not during the enactment. Therefore, no changes on group organizations are possible with this tool.

For the instantiation phase, Hernández-Gonzalo et al propose an IMS LD compliant tool called iCollage [6]. This is a graphical tool for the particularization of role/group structures aiming at facilitating the creation of instances and population of groups. One interesting innovation that this tool features is that groups can be defined during the instantiation phase instead of during edition, allowing the user to adapt group structures to the real contextual situation. However, this tool only provides graphical support for the group population according to the previous 
structures determined during the script edition. Thus, it fails to allow modifications during the script enactment, in which the extrinsic constraints can force changes in the structure planned during the edition process.

Finally, Zarroandia et al proposes a mechanism for the introduction of small variations in the original IMS LD learning flow during the enactment [16]. This tool allows changing some aspects of the activity such as the title, the resources associated or the structure of the learning flow. Nevertheless, the group hierarchies and the roles defined during the edition phase cannot be changed during the enactment.

The main problem of these approaches is that they treat separately the edition from the instantiation and enactment phases. This means that the group structures planned during the edition cannot be adjusted to the contextual situations during the enactment.

\subsection{Considering the intrinsic constraints of two IMS LD CLFPS}

This work proposes a solution for flexibly managing groups of students according to Collaborative Learning Flow Pattern (CLFP) principles when applied to blended learning contexts. For the proposal we adopt: (1) the constrain-based framework proposed by Dillenbourg and Tchounikine as a basis for understanding the flexibility requirements that arise from collaborative learning practices and (2) the IMS LD specification as the de facto standard for our implementations for assuring the interoperability with the current developments and an easier integration with the existing tooling conform to this specification.

The solution is based on a conceptual model developed by the authors in a previous work. This model proposes four factors conditioning the group management in blended learning scenarios [15]: the Pedagogical Method (the activity workflow that defines the groups and role distributions), the Participants (potential and actual people participating in the activity), the History (the unexpected events fruits from the context) and the Space (elements of the space involved in the activity). The first three factors proposed in the model are the basis for identifying the main aspects to be considered when analyzing the requirement of a system for supporting the group management. The Space factor will be considered in future studies. As the Pedagogical Method factor we adopt a CLFP codified with the IMS Learning Design specification.

For addressing the flexibility requirements of the group organization we analyze two particular CLFPs, Jigsaw and TAPPS (Thinking Aloud Pair Problem Solving) by dissociating the constraints intrinsic to the pedagogical design of the script from those induced by the contextual factors. From the analysis, we extract a set of constraints for each of the CLFPs and map them with some of the elements of their IMS LD codification. This mapping leads to a formal representation of the 
educational flexibility requirements. The results define the foundations of a technological architecture based on a notification system for facilitating the adaptation of the CLFPs to the unexpected events arising from the learning context by preserving their main rationale. In the following sections, the analysis of the constraints, their mapping with the IMS LD and the web-based prototype resulting from this proposal are detailed.

\section{Flexibility constraints for TAPPS and Jigsaw}

To study the flexibility requirements for the group management in the Jigsaw and TAPPs CLFPs we follow the definitions given in [7]. We adopt the main indications regarding the group composition and the role distribution along phases for extracting the intrinsic constraints. The aim at selecting these concrete CLFPs is to consider two CLFPs with different levels of complexity in order to understand the effectiveness of using technology for supporting these practices. This section presents the 1) description of both CLFPs, 2) the analysis of the intrinsic constraints regarding the group management, 3) the notification messages proposed in case that these constraints are violated for guiding the users through the best grouping solution according to the actual circumstances and 4) the mapping of the IMS LD codification and these intrinsic constraints.

\subsection{Jigsaw and TAPPS CLFPS}

The Jigsaw CLFP organizes a complex learning flow for a context in which several small groups are facing the study of a lot of information for the resolution of the same problem [7]. The activity flow is structured in three phases: i) a first phase in which an individual or initial group studies a particular subproblem, ii) a second phase in which the students that are involved in the same problem are grouped in Expert groups for exchanging ideas, and iii) a third phase in which the students are grouped in Jigsaw groups formed by one expert in each subproblem to solve the whole problem. It is based on the principle that to solve a complex divisible task collaboratively promotes three main educational benefits: positive interdependence, discussion and individual accountability.

Table I analyzes the intrinsic constraints for the Jigsaw pattern. The intrinsic constraint (a) is related with the minimum number of students with a different expert role necessary for applying this pattern. Since the main script principle is based on the division of the task, applying this script requires, at least, having an enough number of students to define two different expert roles. Otherwise, the system should notify the teacher that the script could not be applied. The constraint (b) regards with the difference between the number of potential (E) and actual (E') students. A non equilibrated number of students per expert group can lead to an inconsistency when forming jigsaw groups, such as having a jigsaw group without 
one of the expert roles. For that reason, a variation on the number of expected students should be notified to advice the teacher that s/he should adjust their jigsaw groups in the next phases. Constraints (c), (d) and (e) have to do with the requirements of jigsaw groups (J). This CLFP defines that the appropriate number of students per jigsaw group is within 4 to 5 . Although the script could be applied with three students per group, the system notifies the teacher that the restrictions imposed by the CLFP are not accomplished (notifications (c) and (d) in the table). Finally, in case of having jigsaw groups without one expert of each type, the teacher is advised that it is necessary to re-adjust the jigsaw groups for reaching the expected learning objectives defined by the script.

TABLE I Intrinsic constraints of CLFP Jigsaw

\begin{tabular}{|c|c|c|}
\hline Intrinsic Constraints & Violations & Notification of the system \\
\hline a) $\# \mathrm{E}>=2$ & $\# \mathrm{E}=1$ & $\begin{array}{l}\text { Not.: You need at least } 2 \text { different ex- } \\
\text { pert groups for applying this pattern. }\end{array}$ \\
\hline $\begin{array}{l}\text { b) EG must be formed by the } \\
\text { same \# of students. The EGs } \\
\text { must be equilibrated. }\end{array}$ & $\# \mathrm{E} \neq \# \mathrm{E}$ & $\begin{array}{l}\text { Not.: Be careful when creating the Jig- } \\
\text { saw groups in the next phase. You } \\
\text { have a non equilibrated group of stu- } \\
\text { dents in each EG. }\end{array}$ \\
\hline $\begin{array}{l}\text { c)\#J in } \mathrm{JG}<=\text { max size JG (by } \\
\text { default) }\end{array}$ & $\# \mathrm{~J}$ in $\mathrm{JG}>\max$ size $\mathrm{JG}$ & $\begin{array}{l}\text { Not.: The number of students in Jig- } \\
\text { saw groups is different than the one } \\
\text { stipulated by the CLFP }\end{array}$ \\
\hline d) $\# \mathbf{J}>$ min size $J G$ (by default) & $\# \mathbf{J}<$ min size $\mathrm{JG}$ & $\begin{array}{l}\text { Not.: The number of students in Jig- } \\
\text { saw groups is different than the one } \\
\text { stipulated by the CLFP. }\end{array}$ \\
\hline $\begin{array}{l}\text { e) } \mathrm{JG} \text { are formed by at least } \\
\text { one } \mathrm{E} \text { from each topic }\end{array}$ & $\mathrm{JG}<\# \mathrm{E}$ de un $\mathrm{EG}$ diferent & $\begin{array}{l}\text { Not.: Your jigsaw groups don't con- } \\
\text { tain members of the different expert } \\
\text { groups. Please, review the proposed } \\
\text { distribution and adapt your groups to } \\
\text { this restriction. }\end{array}$ \\
\hline
\end{tabular}

$\mathrm{E} / \mathrm{E}$ '=\# (potential/actual) students with Expert role $\mathrm{J}=\#$ students with Jigsaw role, EG=Expert Group, JG=Jigsaw Group; T=total students

The TAPPs CLFPs gives the organization for a context in which several students are paired and given a series of problems [7]. Each member of the pair is given a role of Problem Solver and Listener that switches for each problem. The Problem Solver reads aloud and talks through the solution of the problem. The Listener follows the problem solver's steps, catches the errors and asks questions for guiding the problem solver to the solution.

Table II analyzes the intrinsic constraints of the TAPPs pattern. Constraints (a) and (b) regard with the number of students (T) and the roles distribution. Since the script proposes working in pairs, if the number of students is odd, the system should notify the teacher that it is necessary to create a group of three persons and distribute the roles of listener and problem's solver accordingly. A group of three must have only one problem solver at once per phase. Constraint (c) is related 
with the role of the students. In case of having pairs, the role between listener and problem solver switches each phase. However, if there is a group of three, the teacher should control that one of the students in this group repeats the same role in two consecutive phases.

TABLE II Intrinsic constraints of the TAPPS CLFP

Phase 1: Individual or initial group

Intrinsic Constraints

a) $\mathrm{T}$ is pair
Violations

$\mathrm{T}$ is odd
Notification of the system

Distribute the students in pairs and locate the orphan student in one of the groups and assign him the listener role.

Not.: The number of students is odd and we propose you to do one group of three persons.

b)In a $\mathrm{P}$ there should be, at least, There are groups of three persons. Not.: You have one group one L and one PS. of three. Pay attention for the role distribution in this group. Be sure that there is only one problem solver at once per phase.

c) The $\mathrm{P}$ switch roles each phase. If $\mathrm{P}>3,1 \mathrm{PS}$ and 2 Listeners. Not:: You have one group of three. Be sure that one of the members in this In case of having a group of three one student plays the same role in group plays the same role of listeners in two contwo consecutive phases $(\mathrm{N}, \mathrm{N}+1)$

$\mathrm{T}=$ total students in class; $\mathrm{P}=$ pair, $\mathrm{L}=$ Listener; $\mathrm{PS}=$ Problem's solver

\subsection{Representing the intrinsic constraints with IMS LD}

We take as a starting point two CLFPs codified as a Unit of Learning (UoL) in IMS LD that we created with Collage [5, 8] and Recourse [17]. For the UoLs' definitions, we follow the guidelines specified in [7] and we configure them as the minimum units needed for representing the CLFPs in IMS LD. A UoL is composed by a set of resources and an $x \mathrm{ml}$ file called manifest that relates them. We benefit from the manifest definition for extracting the intrinsic constraints defined in tables I and II of the previous section.

The component <imsld: roles> defines the hierarchy of the groups by setting the different roles that will be involved in the activity (Fig. 1). By default, IMS LD 
distinguishes between two types of roles: learners and stuff. Another attribute defines the minimum (min-persons) and the maximum (max-persons) number of persons playing the same role. This corresponds to the size of the groups and gives implicit information about the amount of groups. The last element is create-new. When it is set to "allowed" indicates that it is possible to create occurrences of groups of the same type, i.e. groups of people with the same role.

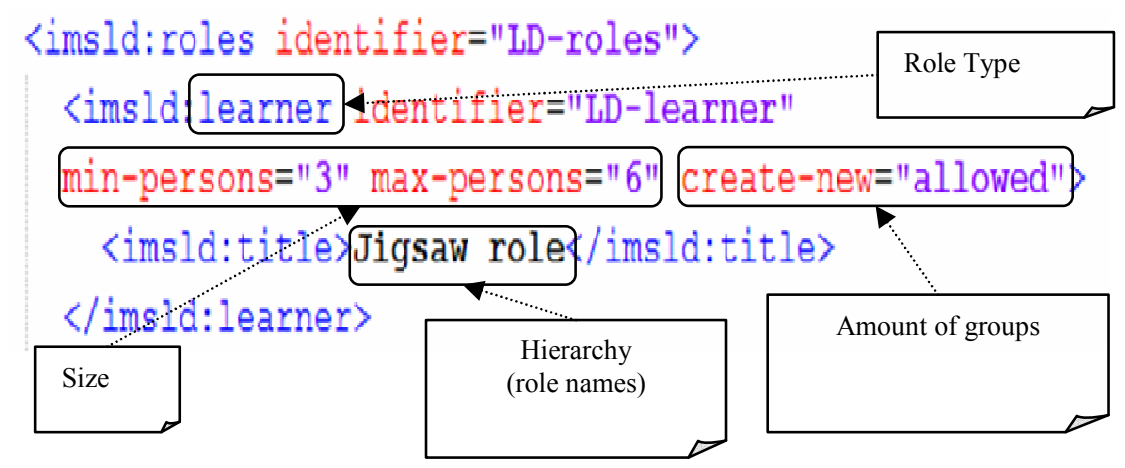

Fig. 1 IMS LD elements of the manifest defining the characteristics of the roles and groups

The learning flow with its activities and the activity-dependent-associations or $d y$ namic formation are defined in the <imsld:method> (Fig. 2). This section defines a set of $\langle$ imsld:act $\rangle$. Each act refers to a sequence of activities defined in the <imsld:activities>, in which are also described the roles taking part in each activity (<imsld: role-part $\rangle$ ).

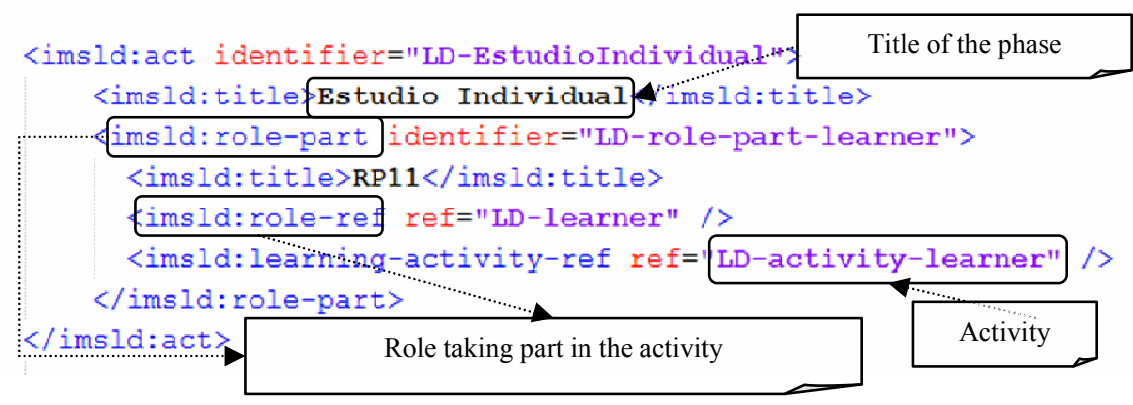

Fig. 2 IMS LD elements from the manifest defining the sequences of activities and the activity dependent associations. 


\section{Supporting flexibility for group management: A web-based tool}

We present here below a prototype as a first effort for supporting group management in blended learning scenarios where CLFPs are applied. This prototype has been designed for the two particular CLPFs Jigsaw and TAPPs taking as a basis the analysis and representation of the intrinsic constraints presented in sections 2 and 3.

\subsection{A web-based application}

We developed a web-based application that distinguishes between a view for the teacher and a view for the learner. The teacher's view includes functionalities for allowing the management of Participants' factor manually or automatically. When using the automatic distribution the system provides always the best possible distribution trying to respect as much as possible the intrinsic constraints. However, the teacher has always the flexibility to change the group distribution proposed (without changing the number of phases or the roles' definition). In case that one constraint is violated, the teacher will be notified but will be always free of leaving the organization as desired. The students view only shows the general group distribution for each phase and the position of the student accessing the system highlighted in another color. The student cannot change any configuration but access to the information stored about his role in other phases.

\subsection{The architecture}

As a basis for the architecture we use three of the factors conditioning the group management to blended learning scenarios defined in [15]: the Pedagogical Method, the Participants and the History (Fig. 3). The Pedagogical Method defines the learning flow of the collaborative activity and it is represented here by a CLFP codified in a UoL conforming to IMS LD. Concretely, the flow of activities and their associations are represented by the elements described in section II, which are parsed from the manifest and codified as the intrinsic constraints in the system according to the tables I and II. The Participants factor is directly associated, in one hand, to the list of potential students that the teacher can upload to the system during the preparation of the group distribution and, on the other hand, to the actual students during the development of the activity. Finally, the History factor stores the information about the group distribution and the new group configurations that occur during the activity development. The unexpected events affecting 
the group composition are stored as extrinsic constraints. A constraints' controller is always listening to the system for notifying the user if any of the intrinsic constraints have been violated. In this case, it will propose an optimal distribution of the participants according to the Pedagogical and the History factors. The system will always propose an alternative, except when the actual number of participant's configuration makes it impossible to satisfy them. In such cases, the system proposes the best alternative or recommends using other CLFPs for this learning scenario. Fig. 3 shows a general picture of the main elements of the system.

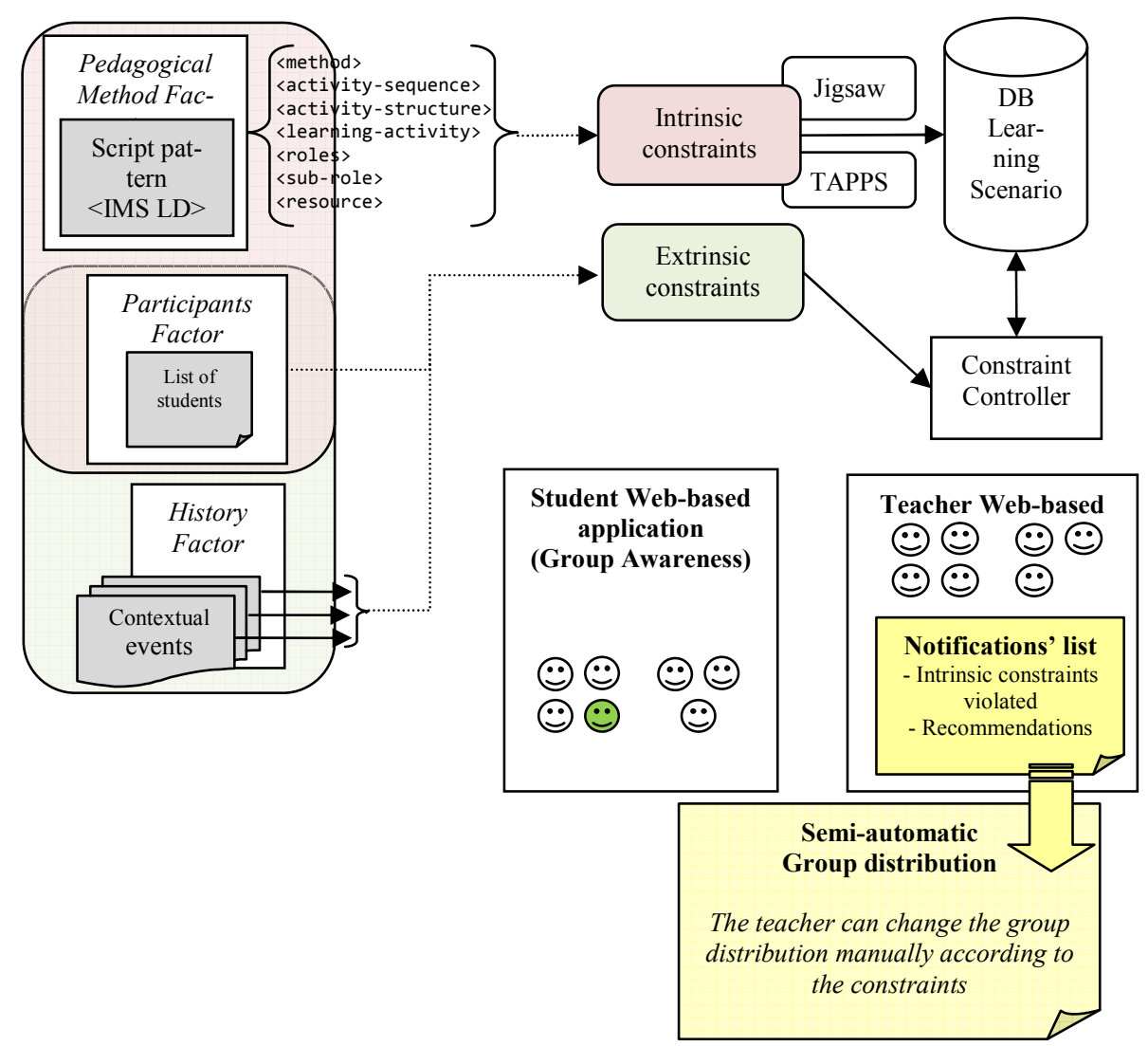

Fig.3 . Schema of the architecture underlying the prototype. The three factors are represented: the pedagogical method, the participants and the History 


\section{Preliminary user study evaluation}

With the aim at obtaining the first evaluation results of the prototype we conducted a preliminary controlled user study. The study focuses on understanding the effectiveness of a tool for flexibly supporting the group management in front of a manual process and indicating in which situations this approach is useful. The main questions of interests were: 1) Do the users find helpful to have a semiautomatic tool for the group management in collaborative activities? 2) Is the tool flexible enough to freely adjust the groups to the unexpected situations? 3) Does the tool support correctly the whole process and in which situations?

\subsection{Description of the user study}

For the user study we prepared two different scenarios: one for the Jigsaw and the other for the TAPPS. Both scenarios described a CLFP in the context of an eLearning course of 13 students. The task of the teacher consisted in organizing the students in groups according to the restriction imposed by the collaborative activity proposed. The scenarios were delivered in a document containing an introduction to the context and the description phase by phase of the CLFP pattern that should be applied. For analyzing the strategies used during the whole process we proposed two different tasks: (1) prepare the group distribution of the potential students from a list according to the requirements of the activity before the class and (2) adapt the groups previously defined to a set of unexpected situations that were described in the scenario as a simulation of the type of events occurring in real educational contexts (i.e. one of the potential students leave the class at the second phase of the activity or a new student joins the class when the activity have already started). In all cases, the restrictions imposed by the CLFP needed to be accomplished. Since the focus of the study was to understand if the tool facilitates the group management in comparison with a manual process we asked the users to perform the two tasks twice, firstly by hand and secondly using the tool. Therefore, the evaluation process was divided in 3 phases: (1) familiarization with the CLFP and the context, (2) group management by hand and (3) group management using the tool.

5 university teachers with 1 to 8 years teaching experience participated in the controlled use case. 2 of them were experts in CSCL practices whereas the other 3 had never prepared a collaborative activity following a CLFP. We assigned the Jigsaw scenario to the 2 experienced users and to 1 inexpert and the TAPPS for the remaining 2. This distribution was focused on comparing the usefulness of the solution in relation to the complexity of the collaborative activity. After a brief explanation of the activity the users started the exercise by performing the group 
distribution manually. In the second phase, we devoted 5 minutes explaining the main functionalities of the tool and the users repeated the exercise using the tool. Since the objective of the evaluation was to understand the whole process and not the design or usability of the prototype, the users were allowed to ask about the functionalities during the experience. Fig. 4 shows the picture of two of the participants of the experience during the two different phases. Two different researchers were recording the observations on how the participants planned their group distributions and their spontaneous comments. During the whole process the users were guided through the different situations by a template with a set of steps. For each step they were asked to explain the strategies followed for the group management and their final students' distribution. All the resulting strategies and distributions were collected. Finally, the users answered a test with close and open questions in which they compared both, the manual and the technologicallysupported processes. Table III summarizes the different data sources considered in the evaluation.
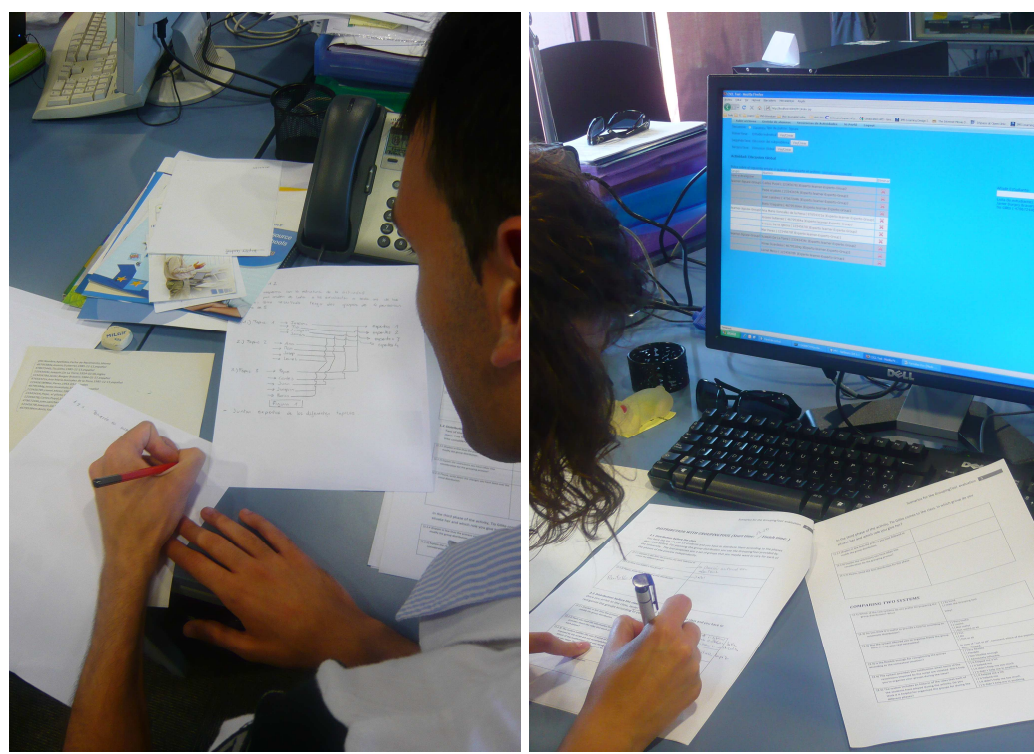

Fig. 4. Teachers participating in the experience. The picture on the left shows the phase in which the activity is carried out by hand and the one on the right corresponds to the phase carried out with the application.

Due to the characteristics of the user study and the objectives of the evaluation, we followed a mixed evaluation method combining and triangulating [1] the qualitative and the quantitative data obtained from the different sources in Table III. As the objective of the evaluation was focused on the process, the qualitative results were used as the main reference for understanding the strategies of the users for solving the unexpected situations and to identify the necessities emerging from this type of practices. 
TABLE III Data sources for the evaluation

\begin{tabular}{|c|c|c|}
\hline Data source & Type of data & Labels \\
\hline \multirow{4}{*}{$\begin{array}{l}\text { Process and out- } \\
\text { comes described by } \\
\text { users in a template }\end{array}$} & Qualitative descriptions and draws. & [Quest-JigsawX] \\
\hline & Qualitative comments and opinions. & [Quest-TAPPSX] \\
\hline & & $\begin{array}{l}\text { Where } \mathrm{X} \text { is the number of the user, } \\
\text { from } 1 \text { to } 5\end{array}$ \\
\hline & $\begin{array}{l}\text { Screenshots of the students' distribu- } \\
\text { tion resulting from the whole process } \\
\text { step by step. }\end{array}$ & $\begin{array}{l}\text { [ToolDistribution-JigsawX] } \\
\text { [ToolDistributionTAPPSX] }\end{array}$ \\
\hline Observations & $\begin{array}{l}\text { Record of direct observations during } \\
\text { the experience by } 2 \text { different re- } \\
\text { searchers. }\end{array}$ & [Observer1] \\
\hline Final questionnaire & $\begin{array}{l}\text { Quantitative ratings and qualitative } \\
\text { opinions comparing the manual and } \\
\text { the technologically-supported proc- } \\
\text { ess. }\end{array}$ & $\begin{array}{l}\text { [Quest-comparison-JigsawX] } \\
\text { [Quest-comparison-TAPPSX] }\end{array}$ \\
\hline
\end{tabular}

\subsection{Results}

To have a general view of the results we answered the main questions of interests by joining the results from the final questionnaire of the Jigsaw and the TAPPS scenarios (Table IV). A detailed analysis of these general results with the qualitative data permits extracting a generic picture of the tool's effectiveness in front of the manual distribution, understanding how helpful is this approach for the users and which the missing requirements are.

Results of question 1 in table IV show that the users found the tool a good support for managing big groups of students in complex collaborative tasks and for having general visualization of the full group distribution. The users performing the more complex activity (Jigsaw) had a better perception of the tool than those doing the simple one (TAPPS). This supports the idea that such type of solutions are helpful in case of having activities with many constraints to be accomplished and a big number of students to organize. As one of the users performing the easiest task said "In small groups of people with few changes it's easier by hand. You don't need to form the groups with the tool. However, for big groups it would be useful." [Quest-Tapps2]. The draws of the users as outcomes from the manual part (see Fig. 5) also evidence the utility of having a graphical support showing the general group distribution. 
TABLE IV Questions of interest and main results achieved in the user study.

\begin{abstract}
Questions laborative activities?
\end{abstract}

\section{Results}

1) Do the users find helpful to *The 3 users that performed the Jigsaw scenario spent an averhave a semi-automatic tool for age of 10 minutes less doing the exercise with the tool than by the group management in col- hand. Whereas the users performing the TAPPS scenario spent 5

2) Is the tool enough flexible to freely adapt the groups to the unexpected situations?

3) Does the tool support correctly the whole process and in which situations? minutes less in average by hand. Nevertheless, the two TAPPS' users commented that it would be very useful in case of having a bigger number of students, like 30 or 50 . More time devoted for familiarization with the tool would decrease the average time spent in the semi-automatic management.

*4 of the users preferred managing the groups using the tool instead of doing it by hand. The user that preferred doing it by hand commented that, in case of having more students s/he would have chosen the tool.

*All the participants considered the tool very useful for managing groups. They mainly highlighted the automatic group distribution functionality and the visualization of full group organization in which the students are labeled with the name of the group they belong to.

*All participants found the tool flexible or very flexible for reorganizing the groups according to the contextual situation. One of the users considered necessary to include the possibility of creating groups whenever $\mathrm{s} /$ he wanted (the tool only included the possibility of creating a new group in the first phase of the activity).

*All participants doing the Jigsaw scenario found that the notifications provided by the tool when a constraint was violated helped them to understand the errors that they need to solve in order to continue the activity correctly. From the users performing the TAPPS scenario one considered the notification system helpful whereas the other one marked that it did not helped him at all. Nevertheless, this last user answered in a previous question that it was helpful to understand the restrictions imposed by the CLFP.

*All participants used the History of the students for confirming that the distribution proposed by the system was correct and to check the role of the students that they needed to re-allocate for adapting the groups to the real context. Only one user from the TAPPS scenario considered the History not very helpful, however, from her/his comments and the observations, it arises that $\mathrm{s} /$ he used it for controlling the role of the students.

With regards to the flexibility of the tool (question 2) for managing groups, the results show that all the users freely change their planned distribution according to the necessities required by the unexpected events. However, they missed the possibility of creating groups at any phase: "I would like to have the possibility of creating new groups" [Quest-Tapps2]. 


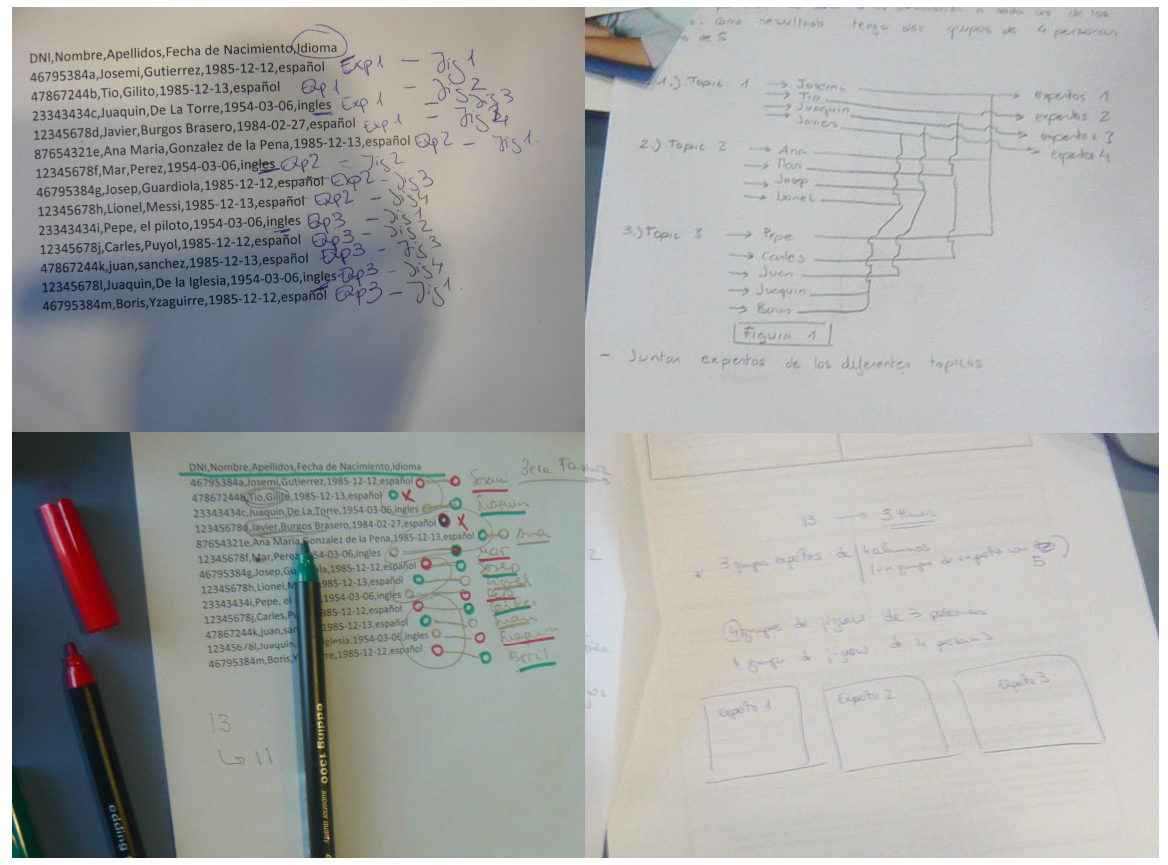

Fig. 5. Draws for organizing the group structures in the Jigsaw scenario.

Finally, the notification and the History of the students serve as a support in the whole process (question 3). All the users re-organized the groups following the notifications provided by the tool and using them as a guide for understanding the constraints that were not fulfilled in their group structure (see Fig. 6 for an example of a screenshot of the process). They used as well the History for checking their final distribution and the list of the students available, thus the potential students that were missing in some of the phases: "I found it very useful to have the list of the students available (although deleted from the activity)" [QuestJigsaw1]. One of the more interesting results was that all users agree with the necessity of adding a button for automatically providing in each phase the best group distribution according to the CLFPs' restrictions.

Some other suggestions for improving the usability of the tool were proposed: 1) change the way that the notifications are showed to the user: "I found the notifications useful just to be sure that everything is ok. However, I will put the warning in yellow and not in red because it seems an error instead of a notification [QuestTabbs2]", 2) use more intuitive systems for manipulating the user in the list and change them from one group to another: "It would be useful to have a drag\&drop functionality to locate the students in the different groups [Quest-Jigsaw1]". 


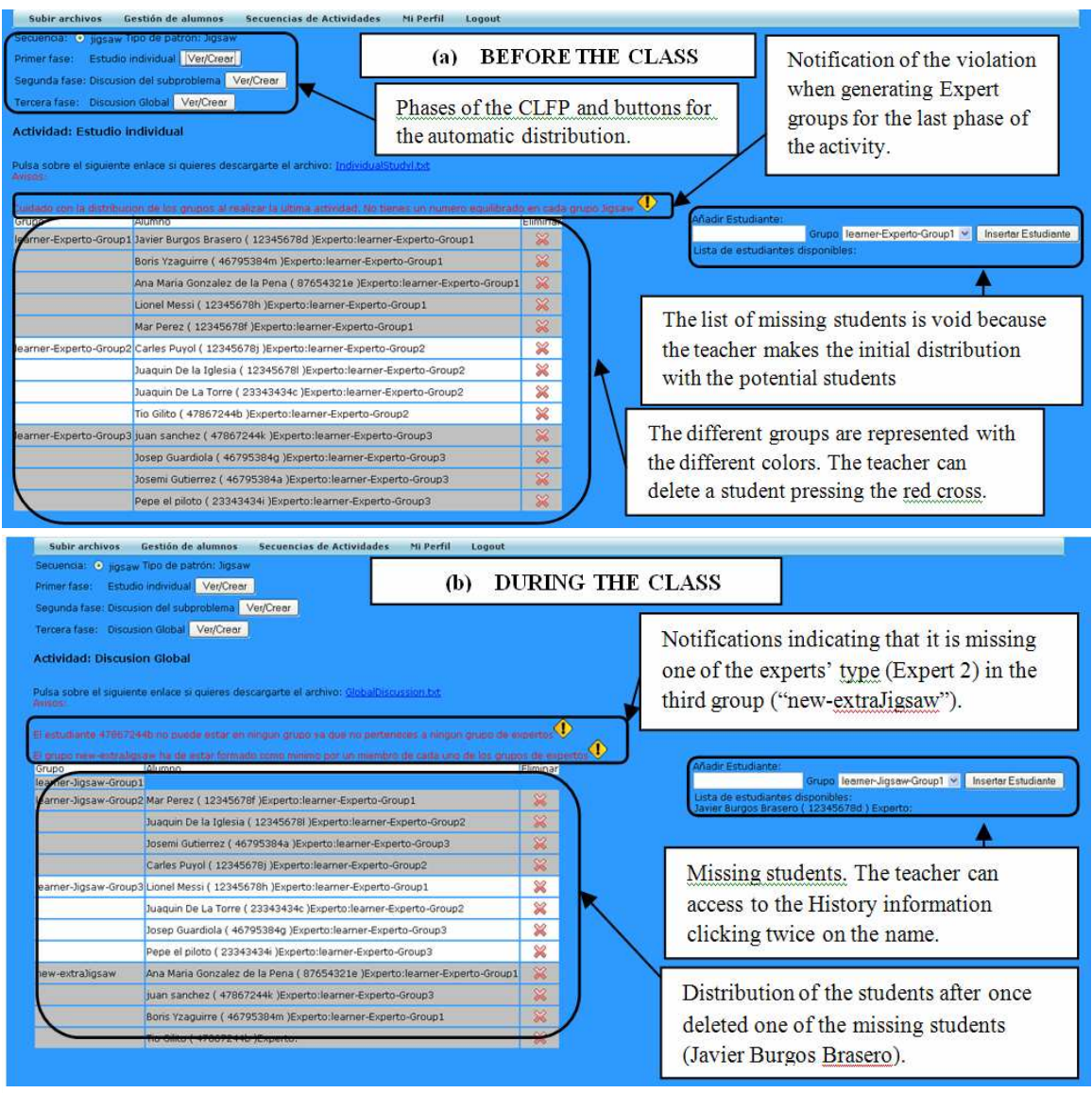

Fig. 6. Screenshots of the prototype. (a) Group distribution before the class. The system proposes the best distribution when clicking on the buttons next to each phase. (b) One of the students is missing and the final distribution is incorrect according to the CLFP's intrinsic constraints. The teacher manually deletes the missing students and attends to the notifications of violations for the final distribution.

\subsection{Future developments}

Future developments are planned to improve the web-based prototype. The first improvement consists on adding the functionalities suggested by the users. According to the users' suggestions, we have already incorporated in the tool an automatic re-distribution button. This new functionality provides the teacher with the best students' distribution according to the intrinsic constraints and the contextual circumstances. We have also changed the color of the notifications from red to yellow for making them less aggressive for the user. 
We also consider extending the tool by providing more sophisticated and formal mechanisms for proposing the best group organization fulfilling the constraints. This requires a further study of the intrinsic constraints for producing a hierarchy ordered depending on whether they are strong (i. e. the number of students is not enough for applying the script) or weak (i. e. although the students' distribution does not fulfill the requirements of the script, the activity can continue without affecting to the final learning outcomes). This classification of constraints would allow at providing more accurate suggestions to the user.

Currently, as an extension of the prototype presented, we are working on functionality for enabling the specification of the Space as a conditioning factor in the design and enactment of the scripting processes. This extension relates with the work of the authors in which the Space, understood as the place where the learning activity occurs and which elements compose it, is considered as a factor influencing the how the groups are distributed for in the design and the enactment of collaborative learning flows [15]. Thus, depending on the characteristics of the physical space where the activity is carried out (with places for working or groups or not), the movement of the students when applying a Jigsaw CLFPs will be possible or not. This physical arrangement will affect on the way students are grouped for the expert groups.

\section{Conclusion and future work}

This work presents a web-based prototype as a solution for flexibly supporting teachers in organizing the groups during the edition according to the principles stipulated by the Jigsaw and TAPPS CLFPs and guiding their re-distribution when unexpected situations occur. The preliminary evaluation results from a controlled user study show that such type of solution is useful mainly in two cases: 1) when performing complex collaborative learning activity in which there are many constraints to control and 2) when preparing activities with a big number of students. The evaluation also evidences that the introduction of a notification system and the History of the students is a good mechanism for guiding the users along the best solution for solving the non-fulfilled constraints. Although a more exhausted evaluation is needed, these preliminary results demonstrate that to consider the intrinsic constraints and the history of the activity facilitates the adjustment of the pre-defined groups to the variability of the context.

As next steps, we aim at performing an evaluation of the tool in a real learning scenario for studying how the notification system and the usability can be improved. We also plan to study the intrinsic constraints of new CLFPs to have a more extensive variety of collaborative situations to enact. The results from the planned evaluation will serve as a basis for improving the notification system by introducing a more sophisticated mechanism for guiding the user in the group adjustments according to the solutions adopted by other practitioners. 


\section{Aknowledgement}

This work was supported in part by the Spanish Ministry of Science and Innovation in the Learn3 project TIN2008-05163/TSI.

\section{References}

[1] Creswell JW (2003) Research design: Qualitative, quantitative, and mixed method approaches. Sage Publications, 2nd ed., Thousand Oaks, London, UK.

[2] Dillenbourg, P (2002) Overscripting CSCL: The risks of blending collaborative learning with instructional design. Three worlds of CSCL. Can we support CSCL?, ed. P.A. Kirschner, Open Universiteit Nederland, , pp. 61-91.

[3] Dillenbourg, P \& Fischer, P (2007). Basics of Computer-Supported Collaborative Learning. Zeitschrift für Berufs- und Wirtschaftspadagogik, vol. 21, pp. 111-130.

[4] P. Dillenbourg and P. Tchounikine (2007) Flexibility in macro-scripts for computersupported collaborative learning. Journal of Computer Assisted Learning Journal of Computer Assisted Learning, vol. 23, no. 1, pp. 1-13.

[5] Hernández-Leo, D, Asensio-Pérez, JI, \& Dimitriadis, Y (2005) Computational Representation of Collaborative Learning Flow Patterns using IMS Learning Design, Journal of Educational Technology and Society, vol. 8, no. 4, pp. 75 .

[6] Hernández, D, Asensio, JI, Dimitriadis, Y, \& Villasclaras, ED (010) Pattern languages for generating CSCL scripts: from a conceptual model to the design of a real situation. In P. Goodyear and S. Retalis (eds), E-learning, design patterns and pattern languages, sense Publishers., pp. 49-64

[7] Hernández-Leo, D, Villasclaras-Fernández, ED, Asensio-Pérez, JI \& Dimitriadis, Y Generating CSCL scripts: From a conceptual model of pattern languages to the design of real scripts, in: E-learning Design Patterns Book, ed. Goodyear P. \& Retalis, S. (In press.)

[8] Hernández-Leo, D, Villasclaras-Fernández, ED, Jorrín-Abellán, IM, Asensio-Pérez, JI, Dimitriadis, Y, Ruiz-Requies, I \& Rubia-Avi, B (2006). Collage, a Collaborative Learning Design Editor Based on Patterns Special Issue on Learning Design, vol. 9, no. 1, pp. 58-71

[9] Hwang, G-J, Yin, P-Y, Hwang, C-W and Tsai, C-C (2008), An Enhanced Genetic Approach to Composing Cooperative Learning Groups for Multiple Grouping Criteria, Educational TEchnology \& Society, vol. 11, no. 1, pp. 148-167.

[10] IMS Learning Design Best Practice and Implementation Guide, http://www.imsglobal.org/learningdesign/ldv1p0/imsld bestv1p0.html [2003a, November 10]

[11] Koper, R \& Olivier, B (2004) Representing the Learning Design of Units of Learning. Educational Technology \& Society, vol. 7, no. 3, pp. 97-111 (2004)

[12] Koper, R and Tattersall, C (2005) Learning Design: A Handbook on Modeling and Delivering Networked Education and Training. Springer Berlin Heidelberg, NY, US

[13] Ounnas, A, Davis, H \& Millard, D (2008). Semantic Web-based Group Formation for Elearning, ESWC, PhD Symposium, Tenerife, Spain, pp. 51-55.

[14] Ounnas, A et al.(2008). A Framework for Semantic Group Formation. IEEE International Conference on Advanced Learning Technologies, ICALT' 08, Spain, pp. 34-38

[15] M. Pérez-Sanagustín, D. Hernández-Leo \& J. Blat. (2009) Conditioning factors for group management in blended learning scenarios, $9^{\text {th }}$ IEEE International Conference on Advanced Learning Technologies, ICALT'09, Riga, 2009, pp. 233-235 
[16] Zarraonandia, T, Dodero, JM \& Fernández, C (2006). Crosscutting Runtime Adaptations of LD Execution. Educational Technology \& Society, vol. 9, no. 1, pp. 123-137.

[17] Recourse, http://www.tencompetence.org/ldauthor [2009, July] 